\title{
Parameter Estimation for the One-Term (Multiterm) Fractional- Order SEIAR Models of Norovirus Outbreak
}

\author{
Tianzeng Li $\mathbb{D}^{1,2}$ Yu Wang $\mathbb{D},{ }^{1}$ and Weiqiu Pan ${ }^{1}$ \\ ${ }^{1}$ School of Mathematics and Statistics, Sichuan University of Science and Engineering, Zigong 643000, China \\ ${ }^{2}$ South Sichuan Center for Applied Mathematics, Yibin 644000, China \\ Correspondence should be addressed to Tianzeng Li; litianzeng27@163.com and Yu Wang; wangyu_813@163.com
}

Received 31 January 2021; Revised 17 April 2021; Accepted 27 May 2021; Published 29 June 2021

Academic Editor: Soheil Salahshour

Copyright (C) 2021 Tianzeng Li et al. This is an open access article distributed under the Creative Commons Attribution License, which permits unrestricted use, distribution, and reproduction in any medium, provided the original work is properly cited.

\begin{abstract}
In the paper, we use the Caputo fractional derivative to consider general single-term and multiterm fractional-order SEIAR models for the outbreak of Norovirus. Then, the inverse problem about parameter estimation for these fractional-order SEIAR models of the Norovirus outbreak is studied firstly. To provide the numerical solution of the single-term (or multiterm) fractional-order nonlinear differential equation, the GMMP scheme and Newton method are introduced. Then, we make use of the modified hybrid Nelder-Mead simplex search and particle swarm optimization (MH-NMSS-PSO) algorithm to obtain the fractional orders and parameters for these fractional-order SEIAR models of Norovirus outbreak. To guarantee the correctness and effectiveness of the methods, the data of a 2007 Norovirus outbreak in a middle school in one city is used as the real data to solve the inverse problem of the parameter estimation. With the new parameters, all numerical studies illustrate that the numerical solutions fit very well with the real data, which reveals that the single-term and multiterm fractional-order SEIAR models of Norovirus outbreak all can predict the number of the infectious people accurately. And it also shows that the GMMP scheme and the MH-NMSS-PSO method are efficient and valid for estimating the parameters of the single-term (or multiterm) fractional-order nonlinear equations. Then, we research the impact of changes in each parameter on the amount of infected humans $I(t)$ when the remaining parameters are unchanged. All results of numerical simulation reveal that the single-term and multiterm fractional-order SEIAR model of Norovirus can provide better results than other models. And we also study the effect of the isolation on different days. The conclusion is obtained that the earlier the isolation is taken, the less the infected people are. Hence, for a fractional-order application in the SEIAR model of Norovirus outbreak, we establish the effective parameter estimation methods.
\end{abstract}

\section{Introduction}

Norovirus is one of the most important pathogens of infectious diarrhea and outbreaks of all ages [1-3]. In the United States, Norovirus gives rise to approximately 21 million cases each year [2], 71 thousand hospitalizations [4], and 8 hundred deaths $[2,5]$. In developing countries, there are frequent outbreaks of medical institutions and schools [6], which have a great impact on the health of residents. The disease is mainly transmitted by means of the fecal-oral route, and the infection dose is very low. Ingestion of 18 viruses at a time can cause infection [7]. Therefore, it is easy to cause transmission, usually by human contact and water or food spread.
School outbreaks can also lead to absenteeism or even suspension of classes, affecting normal teaching order and increasing the burden of family care for children. Therefore, in-depth study of the dynamic characteristics of Norovirus infectious diarrhea outbreaks and the evaluation of the effects of various types of prevention and control measures have important public health significance.

These are difficult to analyze through traditional epidemiological methods, and mathematical modeling has become a crucial research tool for studying the dynamics of infectious diseases $[8,9]$. Based on the natural history of Norovirusinfected diarrhea, the authors established the integer-order SEIAR model of Norovirus transmission in schools. Taking 
an outbreak event in a city in 2007 as an example, the dynamic characteristics of Norovirus were studied and the key prevention and control was quantitatively evaluated. But the results indicate that the match between the number of infections derived by the model and the real data needs to be upgraded further to give expression to the spread of Norovirus, especially the transmission speed before the intervention.

During the last three decades or so, fractional calculus is the focus of almost all dynamic researchers as we often see it in the fields of chemistry physics [10], engineering [11], biology [12, 13], and image processing [14]. Fractional-order derivatives can be called a superb tool which depicts the memory and hereditary properties of all kinds of materials and processes [14, 15]. Fractional differential equations are more adequate than integer-order derivatives to describe some phenomena associated with nonlocality. Hence, the fractional-order systems are also used to deal with some problems of infectious disease [16-18]. The fractional-order derivative can offer a better infectious disease model compared to the integer-order derivative [19]. There are many advantages of fractional differential equation for describing infectious disease. One is that the fractional derivatives are nonlocal operations, i.e., the fractional-order differential equation can establish a powerful tool for integration of memory and hereditary properties of the systems, while the integer-order derivatives are local operations, i.e., the effects are ignored or tough to incorporate in the integer-order differential equation. Another one is that it is precisely because the fractional-order model has one more degree of freedom compared to the integer order when we fit the model to the real data; the fractional-order model is more suitable for studying infectious diseases.

As we know, some parameters are unknown in the fractional-order model, which need to be determined from the real data. Thus, the fractional inverse problem [20] is generated which has aroused worldwide attention on account of the extensive applications [21]. And there are many researches for classical problems $[22,23]$. The two-dimensional nonlinear reaction-subdiffusion equation is solved via the novel compact numerical method [24]. Utilizing a Haar wavelet operational matrix, literature [25] considered an identification method aimed at the fractional-order linear system. In [26], aiming at the one-dimensional fractional diffusion equation, the authors discussed the uniqueness result with regard to deciding the fractional order and the space-dependent diffusion coefficient. Literature [27] proposed a feasible parameter estimation method based on hybrid Nelder-Mead simplex search and particle swarm optimization to perform the curve fitting for the multiterm time-fractional Bloch equations. In [10], the authors applied a parameter fitting approach to the classical monoexponential model, a previously developed anomalous relaxation model, and the extended timefractional relaxation model. They find that the extended time-fractional model is able to fit the experimental data with smaller mean-squared error than the classical monoexponential relaxation model and the anomalous relaxation model. Aside from the parameter estimation method we used in the article, there are also the Bayesian method put forward by
Fan et al. [28], the modified quasiboundary value method [29], and so on. Nowadays, coronavirus is a pandemic that has become a concern for the whole world. Scholars at home and abroad predict [30], analyze [31, 32], and intervene [33] the spread of the coronavirus according to the infectious disease models of coronavirus outbreak. It can be seen that the analysis of infectious diseases based on infectious disease models is of great significance for understanding the trend of infectious diseases, reducing the number of infections, and preventing recurrence.

In this paper, we mainly use the single-term and multiterm fractional-order SEIAR model to describe the outbreak of Norovirus explained by the Caputo fractional derivatives. These SEIAR models are general models with different fractional orders. For the sake of maintaining the same units on both sides of the models, some new parameters are introduced which have different dimensions. This makes certain that both sides of the models have the same dimension. The GMMP schemes are modified to acquire an implicit difference equation which can be identified as nonlinear equations solved by the Newton method. Thus, the problem of numerical solution is derived. There are some other numerical methods, such as q-Homotopy Analysis Transform Method (q-HATM) [34-37], Fractional Euler Method (FEM) [38], Discretized Collocation Method [39], Variational Iteration Method (VIM) [40], and Fractional Natural Decomposition Method (FNDM) [41]. Then, the corresponding inverse problem of parameter estimation is studied by the $\mathrm{MH}$ NMSS-PSO. Using the statistics from the Norovirus outbreak in a middle school in 2007 [42], the fractional-order SEIAR model we proposed can be determined. Based on these new parameters and orders, the numerical results provided by two fractional-order SEIAR models are very closer to the real data.

The content of this paper is introduced as follows: the integer-order Susceptible-Exposed-Infectious/AsymptomaticRemoved (SEIAR) Model of Norovirus outbreak is introduced in Section 2. The fractional derivatives and the single-term fractional-order SEIAR model and its numerical method are introduced in Section 3. In Section 4, the MH-NMSS-PSO algorithm is described. In Section 5, the application of the parameter estimation in the single-term fractional SEIAR model is presented. The multiterm fractional-order SEIAR model is introduced, and the application of the parameter estimation is presented in Section 6. In Section 7, the conclusion is derived.

\section{The Classical SEIAR Model of Norovirus Outbreak}

The SEIAR model is the most common method for studying the dynamic characteristics of some infectious disease, such as Norovirus, influenza, and worm propagation [42, 43]. It is better to divide the total human population $N$ into five subpopulations: $S(t)$ susceptible humans, $E(t)$ exposed humans (infected but not yet fully contagious), $I(t)$ infected humans, $A(t)$ asymptomatic humans, and $R(t)$ recovered (or removed) humans. Hence, the Norovirus classical differential equation is as follows: 


$$
\begin{gathered}
\frac{d S}{d t}=-\beta S(I+k A), \\
\frac{d E}{d t}=\beta S(I+k A)-\mu \omega^{\prime} E-(1-\mu) \omega E, \\
\frac{d I}{d t}=(1-\mu) \omega E-\gamma I, \\
\frac{d A}{d t}=\mu \omega^{\prime} E-\gamma^{\prime} A, \\
\frac{d R}{d t}=\gamma I+\gamma^{\prime} A .
\end{gathered}
$$

In this model (1), the parameters denote different meanings, respectively:

(i) $\beta$ is the probability of transmission per contact

(ii) $k$ is the relative transmissibility of asymptomatic to symptomatic individuals

(iii) $\mu$ is the proportion of asymptomatic individuals

(iv) $1 / \omega$ is the incubation period of the disease

(v) $1 / \omega^{\prime}$ is the latent period of the disease

(vi) $\gamma$ is the removal rate of the infectious individuals

(vii) $\gamma^{\prime}$ is the removal rate of the asymptomatic individuals

In practice, the quarantine measures were applied to all patients $I$, that is, all patients $I$ were quarantined when the health department intervened on the first day. And one patient was quarantined in the subsequent treatment, that is, all patients were quarantined once they were found. Because patient $I$ is quarantined, quarantined patients cannot transmit the disease because they are not in contact with susceptible persons. During isolation, the disease progression of the patient was the same as that of the unquarantined, and the removal rate coefficient from the quarantined remained. But people with latent infections can still contract the disease through person-to-person contact. The differential equation of the quarantine patient model is as follows:

$$
\begin{gathered}
\frac{d S}{d t}=-\beta k S A, \\
\frac{d E}{d t}=\beta k S A-\mu \omega^{\prime} E-(1-\mu) \omega E, \\
\frac{d I}{d t}=(1-\mu) \omega E-\gamma I, \\
\frac{d A}{d t}=\mu \omega^{\prime} E-\gamma^{\prime} A, \\
\frac{d R}{d t}=\gamma I+\gamma^{\prime} A,
\end{gathered}
$$

where the parameters of this model are the same as the model (1).

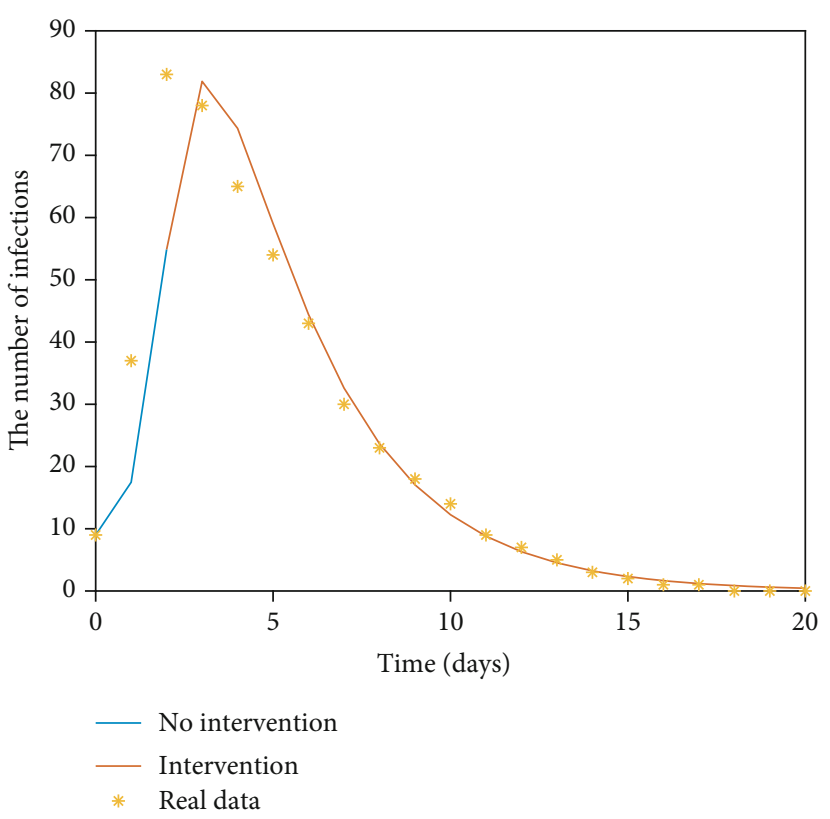

FIgURE 1: Quantity of the Norovirus infections $I(t)$ compare with the numerical results of integer-order SEIAR model (1) obtained by the MGAM method and $g=7.9138$.

In reference [42], they study the data about a Norovirus infectious diarrhea incident reported in a middle school in a city. The information includes the number of people affected, the onset time of all cases, the intervening time of the department of the centers for disease control and prevention, and the preventive and control measures. The details of the outbreak are as follows: on March 8, the department of the centers for disease control and prevention of a city received a telephone report from a middle school, saying that there were more than ten cases of vomiting, abdominal pain, and diarrhea in the school recently. The following case definitions were established: vomiting or diarrhea and other symptoms such as abdominal pain, fever, headache, and dizziness have occurred among the students and staff of the school since March 5. There are 93 classes in 5 grades in the school, with 5225 students and 430 teachers. The number of cases reached a peak on 8 March. After the intervention on $8 \mathrm{March}$, isolation measures were taken. The epidemic situation began to decline gradually.

The authors use the integer-order SEIAR model to predict the number of the infected people. The parameters were selected as $\beta=8.3452 \times 10^{-4}, k=3.9065 \times 10^{-11}, \omega=1, \omega^{\prime}$ $=1, \mu=0.3, \gamma=0.3333$, and $\gamma^{\prime}=0.03846$; the number of infected humans is shown in Figure 1. Based on the results and Figure 1, we find that the root-mean-square error among the numerical solution and the real data is $g=7.9138$, which identifies that the numerical solutions we obtained should be closer to the real data to reflect the spread of Norovirus, especially the speed of the spread before the intervention. Hence, we should find some new model to make the numerical results fit well with the real data. We could predict the change in the number of people infected more correctly. 


\section{Fractional-Order SEIAR Model of Norovirus Outbreak and Its Numerical Method}

3.1. The Definitions and Properties of the Fractional Derivatives. Fractional calculus has a great influence on the development of scientific research $[14,44]$. The most influential definitions for fractional-order derivatives include Riemann-Liouville (R-L), Caputo, and Grünwald-Letnikov (G-L) definition [15].

Definition 1. The fractional integral is introduced as

$$
{ }_{a} D_{t}^{-\alpha} f(t)=\frac{1}{\Gamma(\alpha)} \int_{a}^{t}(t-\tau)^{\alpha-1} f(\tau) d \tau
$$

where the order is $\alpha>0$ and $\Gamma(z)=\int_{0}^{\infty} t^{z-1} e^{-t} d t$ is the gamma function.

Definition 2 (see [24]). The expression of Caputo derivative definition is explained in this way:

${ }_{a}^{C} D_{t}^{\alpha} f(t)={ }_{0} D_{t}^{-(n-\alpha)} \frac{d^{n}}{d t^{n}} f(t)=\frac{1}{\Gamma(n-\alpha)} \int_{a}^{t}(t-\tau)^{n-\alpha-1} f^{(n)}(\tau) d \tau$,

where $n-1<\alpha<n, n \in Z^{+}$.

Definition 3. In 1847, the German mathematician Riemann made further additions on this basis and formed the first more complete definition of fractional calculus-the Riemann-Liouville definition.

${ }_{a}^{R L} D_{t}^{\alpha} f(t)=\frac{d^{n}}{d t^{n}} D_{t}^{-(n-\alpha)} f(t)=\frac{1}{\Gamma(n-\alpha)} \frac{d^{n}}{d t^{n}} \int_{a}^{t}(t-\tau)^{n-\alpha-1} f(\tau) d \tau$,

where $n-1<\alpha<n, n \in Z^{+}$.

Definition 4. The Grünwald-Letnikov derivative is considered as

$$
\begin{aligned}
{ }_{a}^{G L} D_{t}^{\alpha} f(t)= & \lim _{h \longrightarrow 0} h^{-\alpha} \sum_{r=0}^{m}(-1)^{r}\left(\begin{array}{c}
\alpha \\
r
\end{array}\right) f(t-r h) \\
= & \sum_{k=0}^{n-1} \frac{f^{(k)}(0) t^{k-\alpha}}{\Gamma(k+1-\alpha)}+\frac{1}{\Gamma(n-\alpha)} \\
& \cdot \int_{a}^{t}(t-\tau)^{n-\alpha-1} f^{(n)}(\tau) d \tau,
\end{aligned}
$$

where $n-1<\alpha<n$.

Depending on the above definitions, we know that the G$\mathrm{L}$ and R-L fractional derivatives can be transformed into each other; in other words, they are equivalent while the R-L and Caputo definitions are not, in which the difference can be shown as

$$
{ }_{a}^{C} D_{t}^{\alpha} f(t)={ }_{a}^{R L} D_{t}^{\alpha} f(t)-\sum_{k=0}^{n-1} \frac{(t-a)^{k-\alpha} f^{(k)}(a)}{\Gamma(k-\alpha+1)} .
$$

If the initial condition is chose as $f^{(k)}(a)=0, k=0,1, \cdots$, $n-1$, depending on (11), the Caputo and R-L derivatives are equivalent.

Lemma 5. Assumed function $f(t) \in C^{n}[a, T]$, then

$$
{ }_{a}^{C} D_{t}^{\alpha} f(t)={ }_{a}^{R L} D_{t}^{\alpha}\left(f(t)-\sum_{k=0}^{n-1} \frac{(t-a)^{k} f^{k}(a)}{k !}\right),
$$

where $n-1<\alpha \leq n$.

Proof. Suppose

$$
h(t)=f(t)-\sum_{k=0}^{n-1} \frac{(t-a)^{k} f^{(k)}(a)}{k !}
$$

where $h^{(k)}(a)=0, k=0,1, \cdots, n-1$. On the basis of (11), ${ }_{a}^{C} D_{t}^{\alpha} h(t)={ }_{a}^{R L} D_{t}^{\alpha} h(t)$, i.e.,

$$
{ }_{a}^{C} D_{t}^{\alpha} h(t)={ }_{a}^{R L} D_{t}^{\alpha} h(t)={ }_{a}^{R L} D_{t}^{\alpha}\left(f(t)-\sum_{k=0}^{n-1} \frac{(t-a)^{k} f^{(k)}(a)}{k !}\right) .
$$

We can obtain ${ }_{a}^{C} D_{t}^{\alpha}(t-a)^{k}=0$ under the condition of $0 \leq k<\alpha$ by the definition of the Caputo derivative. The left side of equation (14) can be presented as

$$
{ }_{a}^{C} D_{t}^{\alpha} h(t)={ }_{a}^{C} D_{t}^{\alpha} f(t) .
$$

Hence, the result (12) is found.

3.2. Single-Term Fractional-Order SEIAR Model of the Norovirus Outbreak. On the basis of the interorder SEIAR model, the fractional-order SEIAR model and quarantine SEIAR model are as follows:

$$
\begin{aligned}
& \lambda_{\alpha_{10}}{ }^{C} D_{t}^{\alpha_{1}} S=-\beta S(I+k A), \\
& \lambda_{\alpha_{2} 0}{ }^{C} D_{t}^{\alpha_{2}} E=\beta S(I+k A)-\mu \omega^{\prime} E-(1-\mu) \omega E, \\
& \lambda_{\alpha_{30}}{ }^{C} D_{t}^{\alpha_{3}} I=(1-\mu) \omega E-\gamma I \text {, } \\
& \lambda_{\alpha_{40}}{ }^{C} D_{t}^{\alpha_{4}} A=\mu \omega^{\prime} E-\gamma^{\prime} A, \\
& \lambda_{\alpha_{50}}{ }^{C} D_{t}^{\alpha_{5}} R=\gamma I+\gamma^{\prime} A, \\
& \lambda_{\alpha_{10}}{ }^{C} D_{t}^{\alpha_{1}} S=-\beta k S A, \\
& \lambda_{\alpha_{20}}{ }^{C} D_{t}^{\alpha_{2}} E=\beta k S A-\mu \omega^{\prime} E-(1-\mu) \omega E, \\
& \lambda_{\alpha_{30}}{ }^{C} D_{t}^{\alpha_{3}} I=(1-\mu) \omega E-\gamma I,
\end{aligned}
$$




$$
\begin{aligned}
& \lambda_{\alpha_{40}}{ }^{C} D_{t}^{\alpha_{4}} A=\mu \omega^{\prime} E-\gamma^{\prime} A, \\
& \lambda_{\alpha_{50}}{ }^{C} D_{t}^{\alpha_{5}} R=\gamma I+\gamma^{\prime} A,
\end{aligned}
$$

where ${ }_{0}^{C} D_{t}^{\alpha_{i}}$ represents the Caputo fractional derivative with order $\alpha_{i}, i=1,2, \cdots, 5$. The parameters $\beta, k, \mu, \omega, \omega^{\prime}, \gamma, \gamma^{\prime}$ are defined in model (1). In these fractional-order models, we can see that the units on the left of system (16) and (21) maintaining the dimensions of (days) $)^{-\alpha_{i}}$ are not same as the right, in which the dimension is (days) ${ }^{-1}$. If we take the left-hand side of system (16) and (21) by $\lambda_{\alpha_{i}}(i=1,2, \cdots, 5)$ which has the dimension of (days $)^{\alpha_{i}-1}$, then we can have the same units (days) ${ }^{-1}$ on both sides of this system.

3.3. Numerical Result of the Fractional-Order Differential Equation. There are all kinds of numerical methods which have been confirmed to deal with equations (21), covering the Power Series Method [14], the Predictor-Corrector Method [14, 15, 45], the Mellin Transform Method [14], and others. In this article, we adopted the GMMP scheme [46] and Newton method to carry out the equations (21), which are much more efficient than other numerical methods.

Next, we converted equations (21) to the form of

$$
\lambda \odot{ }_{a}^{C} D_{t}^{\alpha} \mathbf{x}(t)=\mathbf{f}(t, \mathbf{x}(t)),
$$

where $\quad \mathbf{x}(t)=(S(t), E(t), I(t), A(t), R(t))^{T} \quad$ and $\quad \lambda=$ $\left(\lambda_{\alpha_{1}}, \lambda_{\alpha_{2}}, \lambda_{\alpha_{3}}, \lambda_{\alpha_{4}}, \lambda_{\alpha_{5}}\right)^{T}$. For the sake of establishing the numerical solution of (21), the uniform grids with $0<\alpha<1$ are applied to discretize in time, that is, $t_{j}=a+j h, j=0,1,2$, $\cdots, N, N h=t-a$. As we know, the R-L and G-L fractional derivatives can be approximated applying this equation:

$$
{ }_{a}^{R L} D_{t}^{\alpha} \mathbf{x}(t)={ }_{a}^{G L} D_{t}^{\alpha} \mathbf{x}(t)=\lim _{h \longrightarrow 0} \frac{1}{h^{\alpha}} \sum_{k=0}^{N} c_{k}^{\alpha} \mathbf{x}\left(t_{N-k}\right) \approx \frac{1}{h^{\alpha}} \sum_{k=0}^{N} c_{k}^{\alpha} \mathbf{x}\left(t_{N-k}\right) .
$$

Similarly, the Caputo fractional derivatives can be explained as

$$
{ }_{a}^{C} D_{t}^{\alpha} \mathbf{x}(t) \approx \frac{1}{h^{\alpha}} \sum_{k=0}^{N} c_{k}^{\alpha}\left(\mathbf{x}\left(t_{N-k}\right)-\sum_{j=0}^{n-1} \frac{(t-a)^{j} \mathbf{x}^{(j)}(a)}{j !}\right),
$$

where,

$$
c_{k}^{\alpha}=(-1)^{k}\left(\begin{array}{l}
\alpha \\
j
\end{array}\right)
$$

are binomial coefficients.

This method appears for the first time in [46] and is widely used by people. In [47], researchers call it the GMMP scheme. Based on it, the numerical solutions of the fractional-order differential equations are revealed. For introducing this method, equation (21) can be written as

$$
\begin{gathered}
\lambda \odot{ }_{a}^{C} D_{t}^{\alpha} \mathbf{x}(t)=\mathbf{f}(t, \mathbf{x}(t)), \quad 0 \leq t \leq T, \\
\mathbf{x}^{(k)}(a)=\mathbf{x}_{0}^{(k)}, \quad k=0,1, \cdots, n-1,
\end{gathered}
$$

where ${ }_{a}^{C} D_{t}^{\alpha}$ represents the Caputo fractional derivative.

It follows from (28) that

$$
\lambda \odot \sum_{k=0}^{N} c_{k}^{\alpha}\left(\mathbf{x}\left(t_{N-k}\right)-\sum_{j=0}^{n-1} \frac{(t-a)^{j} \mathbf{x}^{(j)}(a)}{j !}\right)=h^{\alpha} \mathbf{f}\left(t_{N}, \mathbf{x}\left(t_{N}\right)\right),
$$

i.e.,

$$
\begin{aligned}
\mathbf{x}\left(t_{N}\right)= & h^{\alpha} \oslash \lambda \odot \mathbf{f}\left(t_{N}, \mathbf{x}\left(t_{N}\right)\right)+\sum_{j=0}^{n-1} \frac{(t-a)^{j} \mathbf{x}^{(j)}(a)}{j !} \\
& -\sum_{k=1}^{N} c_{k}^{\alpha}\left(\mathbf{x}\left(t_{N-k}\right)-\sum_{j=0}^{n-1} \frac{(t-a)^{j} \mathbf{x}^{(j)}(a)}{j !}\right) .
\end{aligned}
$$

In particular, if $0<\alpha \leq 1$, (32) should be written as

$$
\mathbf{x}\left(t_{N}\right)=h^{\alpha} \oslash \lambda \odot \mathbf{f}\left(t_{N}, \mathbf{x}\left(t_{N}\right)\right)+\mathbf{x}(a)-\sum_{k=1}^{N} c_{k}^{\alpha}\left(\mathbf{x}\left(t_{N-k}\right)-\mathbf{x}(a)\right) .
$$

In line with the Grünwald-Letnikov formula, the solution form (33) of the fractional-order nonlinear equation has been obtained which can be considered as an equation regarding an unknown variable $\mathbf{x}\left(t_{N}\right)$. We choose the Newton algorithm to gain the value of $\mathbf{x}\left(t_{N}\right)$ via equation (33) which is expressed as

$$
\mathbf{x}_{n+1}=\mathbf{x}_{n}-\mathbf{J}_{\mathbf{F}}\left(\mathbf{x}_{n}\right)^{-1} \mathbf{F}\left(\mathbf{x}_{n}\right), \quad n=0,1,2, \cdots,
$$

where $\mathbf{J}_{\mathbf{F}}\left(\mathbf{x}_{n}\right)$ is the Jacobian matrix at $\mathbf{x}_{n}$. The LU factorization of $\mathbf{J}_{\mathbf{F}}\left(\mathbf{x}_{n}\right)$ can be used to solve the above equations. It is worth mentioning that any other factorization can be presented as well such as QR or Cholesky. Firstly, we should input the initial iterate $\mathbf{x}_{0}$, the nonlinear map $\mathbf{F}$, and termination tolerances $\varepsilon$ into the program.

Step 1. Compute and factor the Jacobian matrix $\mathbf{J}_{\mathbf{F}}\left(x_{0}\right)=\mathbf{L U}$.

Step 2. Solve the linear equation LUs $=-\mathbf{F}\left(\mathbf{x}_{0}\right), \mathbf{x}=\mathbf{s}+\mathbf{x}_{0}$.

Step 3. While $\mathbf{x}$ satisfies the condition $\left\|\mathbf{x}-\mathbf{x}_{0}\right\|>\varepsilon$, then LU

(3a) Let $\mathbf{x}_{0}=\mathbf{x}$, then factor the Jacobian matrix $\mathbf{J}_{\mathbf{F}}\left(\mathbf{x}_{0}\right)=$

(3b) Solve the linear equation LUs $=-\mathbf{F}\left(\mathbf{x}_{0}\right), \mathbf{x}=\mathbf{s}+\mathbf{x}_{0}$

(3c) Compute and evaluate $\left\|\mathbf{x}-\mathbf{x}_{0}\right\|$. If $\left\|\mathbf{x}-\mathbf{x}_{0}\right\|>\varepsilon$, goto step (3a)

Then, we can get the output $\mathbf{x}=\mathbf{x}\left(t_{N}\right)$, i.e., the solution of equation (33). 


\section{The Technique for Obtaining the Parameters of Fractional-Order Nonlinear Systems}

In order for the numerical solution presented by the fractional-order model to be closer to the real number of infected people, we must estimate and correct the original parameters. Regarding the unknown parameters, this problem will become more difficult in the dilemma of the bounded parameters and highly nonlinear on function $f$. Next, a technique is proposed for estimating parameters. The system (26) can be described as the model.

$$
\begin{gathered}
\lambda \odot{ }_{a}^{C} D_{t}^{\alpha} \mathbf{x}(t)=\mathbf{f}(t, \mathbf{x}(t), \mathbf{P}), \quad 0 \leq t \leq T, \\
\mathbf{x}^{(k)}(a)=\mathbf{x}_{0}^{(k)}, \quad k=0,1, \cdots, n-1,
\end{gathered}
$$

where $\mathbf{x}=\left(x_{1}, x_{2}, \cdots, x_{n}\right)^{T}$ are state variables, $\mathbf{f}=$ $\left(f_{1}, f_{2}, \cdots, f_{n}\right)^{T}$ are $n$-dimensional vector functions, and every $f_{i}(i=1,2, \cdots, n)$ represent uncertain parameters $\mathbf{P}=$ $\left(p_{1}, p_{2}, \cdots, p_{m}\right)^{T}$ and $m$ is the amount of parameters.

As we know, many researches adopted the NMSS method [48] and the PSO [49] method to identify parameters. The NMSS concentrates on "exploitation" and PSO concentrates on "exploration." We can distinguish these two methods according to the following two points. One is how to choose the initial point. The initial point of the former is predetermined while the latter is a collection of random points. Second, it depends on the conditions and direction of the proceeding steps. The former moves to points with better function values while the latter moves from points with the worst performance. The MH-NMSS-PSO method has been applied to estimate parameters for integer and fractional models, which fully combines the merits of the NMSS and PSO methods. The idea of combining the NMSS and PSO methods is applied not only to parameter estimation [5052] but also to engineering design [53-55], image processing [56], and economic [57].

In the following, in this paper, we need to find the optimal parameters to make the numerical solution of the fractional-order Norovirus system as close as possible to the number of people infected with Norovirus adopting the MH-NMSS-PSO algorithm. Its steps are as follows.

We suppose that $\mathbf{P}=\left(p_{1}, p_{2}, \cdots, p_{m}\right)^{T} \in \Omega$, where $\Omega=$ $\left[p_{1}^{(\min )}, p_{1}^{(\max )}\right] \times\left[p_{2}^{(\min )}, p_{2}^{(\mathrm{d})}\right] \times \cdots \times\left[p_{m}^{(\min )}, p_{m}^{(\max )}\right] \quad$ is a bounded domain. Let $x\left(t_{j}\right)$ be one of numerical solutions of equation (35) with the given parameters $\mathbf{P}=\left(p_{1}, p_{2}, \cdots, p_{m}\right)$ $\in \Omega$ (such as $I(t)$ ); this result cannot be used as our final data. What we want is the approximation of the unknown parameter vector $\left(p_{1}^{*}, p_{2}^{*}, \cdots, p_{m}^{*}\right)$ determined by the root-meansquare error function (RMSEF):

$$
g\left(\mathbf{P}^{*}\right)=\min _{\mathbf{P} \in D} g(\mathbf{P})=\min _{p \in D} \sqrt{\frac{\sum_{j=0}^{N}\left(\left(x\left(t_{j}\right)-x_{j}\right)\right)^{2}}{N+1}},
$$

where $x_{j}$ are real data.
A potential global minimum $g\left(\mathbf{P}^{*}\right)$ in equation (37) with the parameters $\mathbf{P} \in \Omega$ will be confirmed by the MH-NMSSPSO method. It initiates with $3 m+1$ initial particles consisting of two parts. First of all, the predetermined points are derived to organize an initial simplex with $m+1$ particles which is used in the NMSS method, and the $2 \mathrm{~m}$ particles are optionally created with the help of the PSO method. Then, we sorted the total $3 m+1$ particles from smallest to largest via the function values $g(\mathbf{P})$ in equation (37). In the following, the NMSS method will deal with the best $m+1$ particles while the task of adjusting the last $2 m$ particles is taken care of by the PSO method.

Specifically, it can be divided into the following points.

Step 1. Initialization. Produce a population with size $3 m+1$.

For the minimization of the functions $g(\mathbf{P})$ of $m$ variables (unknown parameters), create $m+1$ vertex points $\mathbf{P}_{i}=\left(p_{1, i}\right.$ $\left., p_{2, i}, \cdots, p_{m, i}\right) \in D,(i=1,2, \cdots, m+1)$ to form an initial $m$ -dimensional simplex, i.e.,

$$
\mathbf{P}_{i}=\left(p_{1, i}, p_{2, i}, \cdots, p_{m, i}\right) \in D, \quad i=1,2, \cdots, m+1,
$$

where

$$
\begin{array}{r}
p_{j, i}=p_{j}^{(\min )}+\frac{(i-1) \times\left(p_{j}^{(\max )}-p_{j}^{(\min )}\right)}{m+1}, \\
j=1,2, \cdots, m, i=1,2, \cdots, m+1 .
\end{array}
$$

Two particles are randomly generated in each dimension for the PSO part:

$$
\mathbf{P}_{i}=\left(p_{1, i}, p_{2, i}, \cdots, p_{m, i}\right) \in D, \quad i=m+2, \cdots, 3 m+1,
$$

where

$$
\begin{aligned}
p_{j, i}=p_{j}^{(\min )}+\operatorname{Rand} \times\left(p_{j}^{(\max )}-p_{j}^{(\min )}\right), \\
\quad j=1,2, \cdots, m, i=m+2, \cdots, 3 m+1,
\end{aligned}
$$

and Rand is a random number in the range $(0,1)$. The particle's initial velocities in each dimension are selected by

$V_{j, i}=\frac{V_{j}^{(\max )}-V_{j}^{(\min )}}{L_{j}}, \quad j=1,2, \cdots, m, i=m+2, \cdots, 3 m+1$,

where $L_{j}(j=1,2, \cdots, m)$ are selected integers.

Step 2. For num $=1: N_{\text {iter }}$ do.

(2a) Evaluation ranking: the function value $g(\mathbf{P})$ of each particle is different. Rank them by evaluating the objective function values

$$
g\left(\mathbf{P}_{1}\right) \leq g\left(\mathbf{P}_{2}\right) \leq \cdots \leq g\left(\mathbf{P}_{3 m+1}\right)
$$

(2b) The NMSS method is applied to the best $m+1$ 
particles and the $(m+1)$ th particle. Calculate $\mathbf{P}_{O}$, the center of gravity of all points except $\mathbf{P}_{m+1}$ :

$$
\mathbf{P}_{O}=\left(p_{1, O}, p_{2, O}, \cdots, p_{m, O}\right) \in D
$$

where $p_{j, O}=\sum_{i=1}^{m} p_{j, i} / m, j=1,2, \cdots, m$. And the reflected point

$$
\mathbf{P}_{r}=(1+\alpha) \mathbf{P}_{O}-\alpha \mathbf{P}_{m+1},
$$

where $\alpha$ is the reflection coefficient $(\alpha>0)$. Nelder and Mead suggest using $\alpha=1$ [48]. Then, there are three cases in the following:

Case 1: $g\left(\mathbf{P}_{1}\right) \leq g\left(\mathbf{P}_{r}\right) \leq g\left(\mathbf{P}_{m}\right)$, then $\mathbf{P}_{r}$ replaces $\mathbf{P}_{m+1}$.

Case 2: $g\left(\mathbf{P}_{r}\right) \leq g\left(\mathbf{P}_{1}\right)$, then compute the expanded point.

$$
\mathbf{P}_{e}=\gamma \mathbf{P}_{r}+(1-\gamma) \mathbf{P}_{O}
$$

If $\mathbf{g}\left(P_{e}\right) \leq g\left(\mathbf{P}_{1}\right), \mathbf{P}_{e}$ replaces $\mathbf{P}_{m+1}$; otherwise $\mathbf{P}_{r}$ replaces $\mathbf{P}_{m+1}$. Nelder and Mead suggest using $\gamma=2$ [48].

Case 3: $g\left(\mathbf{P}_{r}\right) \geq g\left(\mathbf{P}_{m}\right)$, if $g\left(\mathbf{P}_{r}\right) \leq g\left(\mathbf{P}_{m+1}\right), \mathbf{P}_{r}$ replaces $\mathbf{P}_{m+1}$. Compute the contracted point:

$$
\mathbf{P}_{c}=\beta \mathbf{P}_{m+1}+(1-\beta) \mathbf{P}_{c} .
$$

If $g\left(\mathbf{P}_{c}\right) \leq g\left(\mathbf{P}_{m+1}\right), \mathbf{P}_{r}$ replaces $\mathbf{P}_{m+1}$; otherwise, let

$$
\mathbf{P}_{i}=\sigma \mathbf{P}_{i}+(1-\sigma) \mathbf{P}_{1}, \quad i=1,2, \cdots, m+1 .
$$

Nelder and Mead suggest using $\beta=0.5$ and $\sigma=0.5$ [48].

(2c) The PSO method is applied to adjust the last $2 \mathrm{~m}$ particles.

(2d) Discriminate the stopping criterion: if $S_{c}<\varepsilon$, the loop will stop. Let the criterion be defined by

$$
S_{c}=\left[\sum_{i=1}^{m+1} \frac{\left(\bar{g}-\sqrt{g_{i}}\right)^{2}}{m+1}\right]^{1 / 2},
$$

where $\quad \bar{g}=\sum_{i=1}^{m+1}\left(g_{i}^{*} / m+1\right) \quad$ and $\quad g_{i}^{*}=\sqrt{g_{i}}=$ $\sqrt{g_{i}\left(p_{1}, p_{2}, \cdots, p_{m}\right)}$.

Step 3. Output the best estimated parameter values $\mathbf{P}=$ $\left(p_{1}, p_{2}, \cdots, p_{m}\right)$.

Remark 6. The Nelder-Mead simplex search (NMSS) is likely to be trapped in local optima rather than global optima and the particle swarm optimization (PSO) algorithm has a slow convergence rate and low balance between exploration and exploitation. Interestingly, the modified hybrid NelderMead simplex search and particle swarm optimization (MH-NMSS-PSO) algorithm has been demonstrated to outperform both in terms of solution quality and convergence rate. Although the optimal parameters obtained by the new method can provide a solution very close to the real data, it takes a long time to run, and sometimes, the number of iterations is relatively high in order to obtain the optimal parameters.

\section{The Numerical Simulations of the Fractional- Order SEIAR Model}

In this chapter, we will make use of the MH-NMSS-PSO algorithm to obtain the ideal parameters to make the numerical results of the Norovirus system (16) as close as possible to the number of people infected with Norovirus. For exhibiting the parameters of the fractional-order SEIAR model, the real data of a 2007 Norovirus outbreak in a middle school given by [42] is used to carry out the inverse problem of parameter estimation. Each of the parameters in the SEIAR model (16) has its particular biological meaning. Then, each parameter has a corresponding value range. The choice of parameter intervals to narrow the target value is crucial for the result. Hence, based on these ranges, the intervals and initial velocities are chose in this way:

$0 \leq \lambda_{\alpha_{i}}=p_{i} \leq 2, \quad i=1,2, \cdots, 5,0 \leq \alpha_{i}=p_{i+5} \leq 1, \quad i=1,2, \cdots, 5$, $1 \times 10^{-4} \leq \beta=p_{11} \leq 1 \times 10^{-3}, \quad 1 \times 10^{-11} \leq k=p_{12} \leq 1 \times 10^{-10}$, $0.01 \leq \mu=p_{13} \leq 0.5, \quad 0.5 \leq \omega=p_{14} \leq 2$, $0.5 \leq \omega^{\prime}=p_{14} \leq 2, \quad 0.17 \leq \gamma=p_{15} \leq 1$, $0.01 \leq \gamma^{\prime}=p_{16} \leq 0.09$

$$
V_{i}= \begin{cases}0.02, & i=1,2, \cdots, 5, \\ 0.01, & i=6,7, \cdots, 10 \\ 1 \times 10^{-4}, & i=11 \\ 1 \times 10^{-11}, & i=12, \\ 0.025, & i=13,14 \\ 0.01, & i=15,16 .\end{cases}
$$

Based on the potential global minimum of $g(\mathbf{P})$, the unknown parameter $\mathbf{P}^{*}$ is obtained

$$
\begin{gathered}
\lambda_{a_{1}}=0.35201, \quad \lambda_{a_{2}}=0.36513, \quad \lambda_{a_{3}}=1.99264, \quad \lambda_{a_{4}}=1.27217, \\
\lambda_{a_{5}}=1.04215, \quad a_{1}=0.81292, \quad a_{2}=0.99693, \quad a_{3}=0.99981, \\
a_{4}=0.93916, \quad a_{5}=0.91295, \quad \beta=8.33565 \times 10^{-4}, \quad k=1.02143 \times 10^{-11}, \\
p=0.21034, \quad \omega=2.00000, \quad \omega=1.99679, \quad \gamma=0.50863, \gamma^{\prime}=0.08663,
\end{gathered}
$$

with $g=3.1922$. As seen in Figure 2, both the two methods are capable of effectively solving inverse problems for the fractional-order Norovirus system.

By observing how the parameters influence the variety of infected people $I(t)$ when other parameters are unchanged, we can obtain the conclusion when $\lambda_{\alpha_{4}}, \lambda_{\alpha_{5}}, \alpha_{4}, \alpha_{5}, k, \gamma^{\prime}$ changed; the number of infected people $I(t)$ basically did not change while other parameters have a certain impact on $I(t)$. And the impacts of other orders and parameters are 


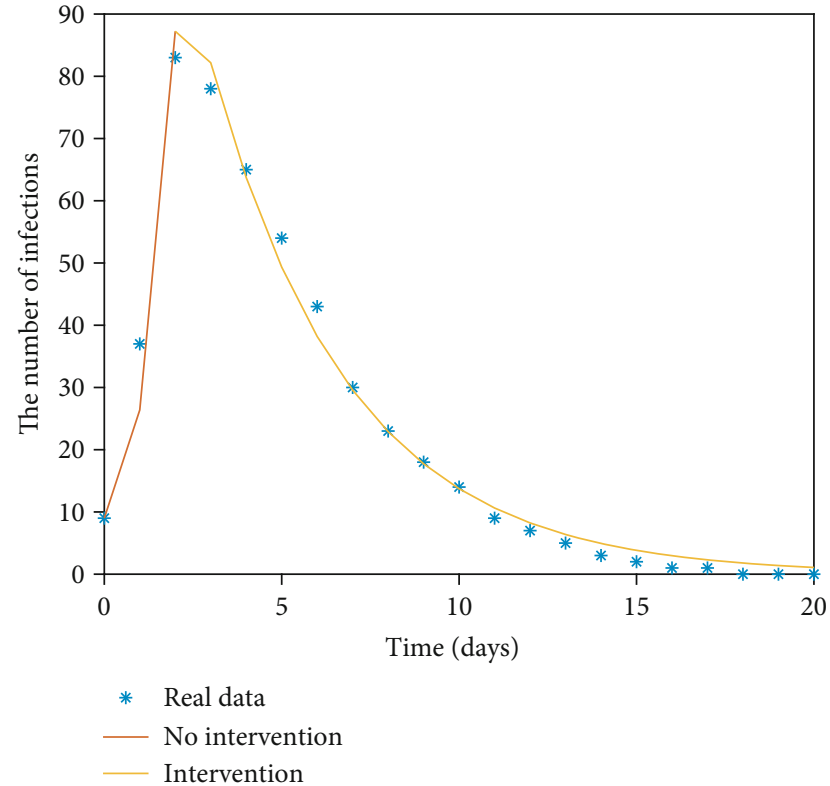

FIGURE 2: Quantity of the Norovirus infections $I(t)$ compared with the numerical results of one-term fractional-order SEIAR model (16) obtained by the MGAM method and $g=3.1922$.

given in Figures 3and 4, which show that the parameter $\mathbf{P}^{*}$ we estimated is indeed the ideal parameter.

\section{The Numerical Simulations of the Multiterm Fractional-Order SEIAR Model}

6.1. The Expression of Numerical Solution. As we know, the multiterm fractional-order differential equations have acted with a significative role in revealing the behaviour of real materials, particularly in the area of the viscoelastic mechanics. In the recent years, some studies have shown that it is more suitable for the study of epidemic models than the integer-order derivative $[10,27]$. In the following, the three-term fractional-order SEIAR model and quarantine SEIAR model of the Norovirus outbreak are proposed as follows:

$$
\begin{gathered}
{ }_{0} D_{t}^{\alpha_{1}, \alpha_{2}, \alpha_{0}} S(t)=-\beta S(I+k A), \\
{ }_{0} D_{t}^{\alpha_{1}, \alpha_{2}, \alpha_{0}} E(t)=\beta S(I+k A)-\mu \omega^{\prime} E-(1-\mu) \omega E, \\
{ }_{0} D_{t}^{\alpha_{1}, \alpha_{2}, \alpha_{0}} I(t)=(1-\mu) \omega E-\gamma I, \\
{ }_{0} D_{t}^{\alpha_{1}, \alpha_{2}, \alpha_{0}} A(t)=\mu \omega^{\prime} E-\gamma^{\prime} A, \\
{ }_{0} D_{t}^{\alpha_{1}, \alpha_{2}, \alpha_{0}} R(t)=\gamma I+\gamma^{\prime} A, \\
{ }_{0} D_{t}^{\alpha_{1}, \alpha_{2}, \alpha_{0}} S(t)=-\beta k S A, \\
{ }_{0} D_{t}^{\alpha_{1}, \alpha_{2}, \alpha_{0}} E(t)=\beta k S A-\mu \omega^{\prime} E-(1-\mu) \omega E, \\
{ }_{0} D_{t}^{\alpha_{1}, \alpha_{2}, \alpha_{0}} I(t)=(1-\mu) \omega E-\gamma I, \\
{ }_{0} D_{t}^{\alpha_{1}, \alpha_{2}, \alpha_{0}} A(t)=\mu \omega^{\prime} E-\gamma^{\prime} A,
\end{gathered}
$$

$$
{ }_{0} D_{t}^{\alpha_{1}, \alpha_{2}, \alpha_{0}} R(t)=\gamma I+\gamma^{\prime} A,
$$

where ${ }_{0} D_{t}^{\alpha_{1}, \alpha_{2}, \alpha_{0}}=\lambda_{1 \cdot{ }_{0}} D_{t}^{\alpha_{1}}+\lambda_{2 \cdot 0} D_{t}^{\alpha_{2}}+\lambda_{0}{ }_{0} D_{t}^{\alpha_{0}}$, and the other parameters in the equation are defined as model (1). However, we can see that the units on the left of equations (52) and (55) maintaining the dimensions of (days) ${ }^{-\alpha_{i}}$ are not same as those on the right in which the dimensions are $(\text { days })^{-1}$. If we take the left-hand side of equations (52) and (55), by $\lambda_{i}(i=0,1,2)$ in which the dimension is (days) $)^{\alpha_{i}-1}(i$ $=0,1,2)$, then we can have the same unit $(\text { days })^{-1}$ to the equations.

The GMMP scheme $[46,47]$ is also adopted to express the numerical solutions of systems (52) and (55). For convenience, the uniform grids $t_{j}=j h, j=0,1,2, \cdots, n$, and $n h=t$ are applied to discretize in time. We converted equations (52) and (55) to the form of

$$
{ }_{a}^{C} D_{t}^{\alpha_{1}, \cdots, \alpha_{r}, \alpha_{0}} \mathbf{x}(t)=\mathbf{f}(t, \mathbf{x}(t)), \quad 0 \leq t \leq T,
$$

where $\mathbf{x}(t)=(S(t), E(t), I(t), A(t), R(t))^{T}$, and $0<\alpha_{1}<\cdots<$ $\alpha_{r}<\alpha_{0}=1$.

Firstly, the fractional derivative can be discretized by equation (52) as follows:

$$
{ }_{0}^{C} D_{t}^{\alpha_{i}} \mathbf{x}\left(t_{n}\right)=\frac{1}{h^{\alpha_{i}}} \sum_{k=0}^{n} c_{k}^{\alpha_{i}}\left(\mathbf{x}\left(t_{n-k}\right)-\mathbf{x}(0)\right), \quad i=1,2, \cdots, r,
$$

where

$$
c_{k}^{\alpha_{i}}=(-1)^{k}\left(\begin{array}{c}
\alpha_{i} \\
j
\end{array}\right)
$$

are binomial coefficients. When $\alpha_{0}=1$, we obtain

$$
{ }_{0}^{C} D_{t}^{\alpha_{0}} \mathbf{x}\left(t_{n}\right)=\frac{d \mathbf{x}\left(t_{n}\right)}{d t}=\frac{\mathbf{x}\left(t_{n}\right)-\mathbf{x}\left(t_{n-1}\right)}{h}+O(h) .
$$

From equations (62) and (65), we can obtain that

$$
\begin{aligned}
{ }_{0}^{C} D_{t}^{\alpha_{1}, \cdots, \alpha_{r}, \alpha_{0}} \mathbf{x}\left(t_{n}\right) & =\sum_{i=1}^{r} \frac{\lambda_{i}}{h^{\alpha_{i}}} \sum_{k=0}^{n} c_{k}^{\alpha_{i}}\left(\mathbf{x}\left(t_{n-k}\right)-\mathbf{x}(0)\right)+\frac{1}{h}\left(\mathbf{x}\left(t_{n}\right)-\mathbf{x}\left(t_{n-1}\right)\right) \\
& =\sum_{k=0}^{n} \sum_{i=1}^{r} \frac{\lambda_{i}}{h^{\alpha_{i}}} c_{k}^{\alpha_{i}}\left(\mathbf{x}\left(t_{n-k}\right)-\mathbf{x}(0)\right)+\frac{1}{h}\left(\mathbf{x}\left(t_{n}\right)-\mathbf{x}\left(t_{n-1}\right)\right) \\
& =\sum_{k=0}^{n}\left(\sum_{i=1}^{r} \frac{\lambda_{i}}{h^{\alpha_{i}}} c_{k}^{\alpha_{i}}\right)\left(\mathbf{x}\left(t_{n-k}\right)-\mathbf{x}(0)\right)+\frac{1}{h}\left(\mathbf{x}\left(t_{n}\right)-\mathbf{x}\left(t_{n-1}\right)\right) \\
& =\sum_{k=0}^{n} B_{k}^{n}\left(\mathbf{x}\left(t_{n-k}\right)-\mathbf{x}(0)\right)+\frac{1}{h}\left(\mathbf{x}\left(t_{n}\right)-\mathbf{x}\left(t_{n-1}\right)\right),
\end{aligned}
$$

where $B_{k}^{n}=\sum_{i=1}^{r}\left(\lambda_{i} / h^{\alpha_{i}}\right) c_{k}^{\alpha_{i}}$.

The discrete scheme of equations (52) is given in the following:

$$
{ }_{0}^{C} D_{t}^{\alpha_{1}, \cdots, \alpha_{r}, \alpha_{0}} \mathbf{x}\left(t_{n}\right)=\mathbf{f}\left(t_{n}, \mathbf{x}\left(t_{n}\right)\right) .
$$



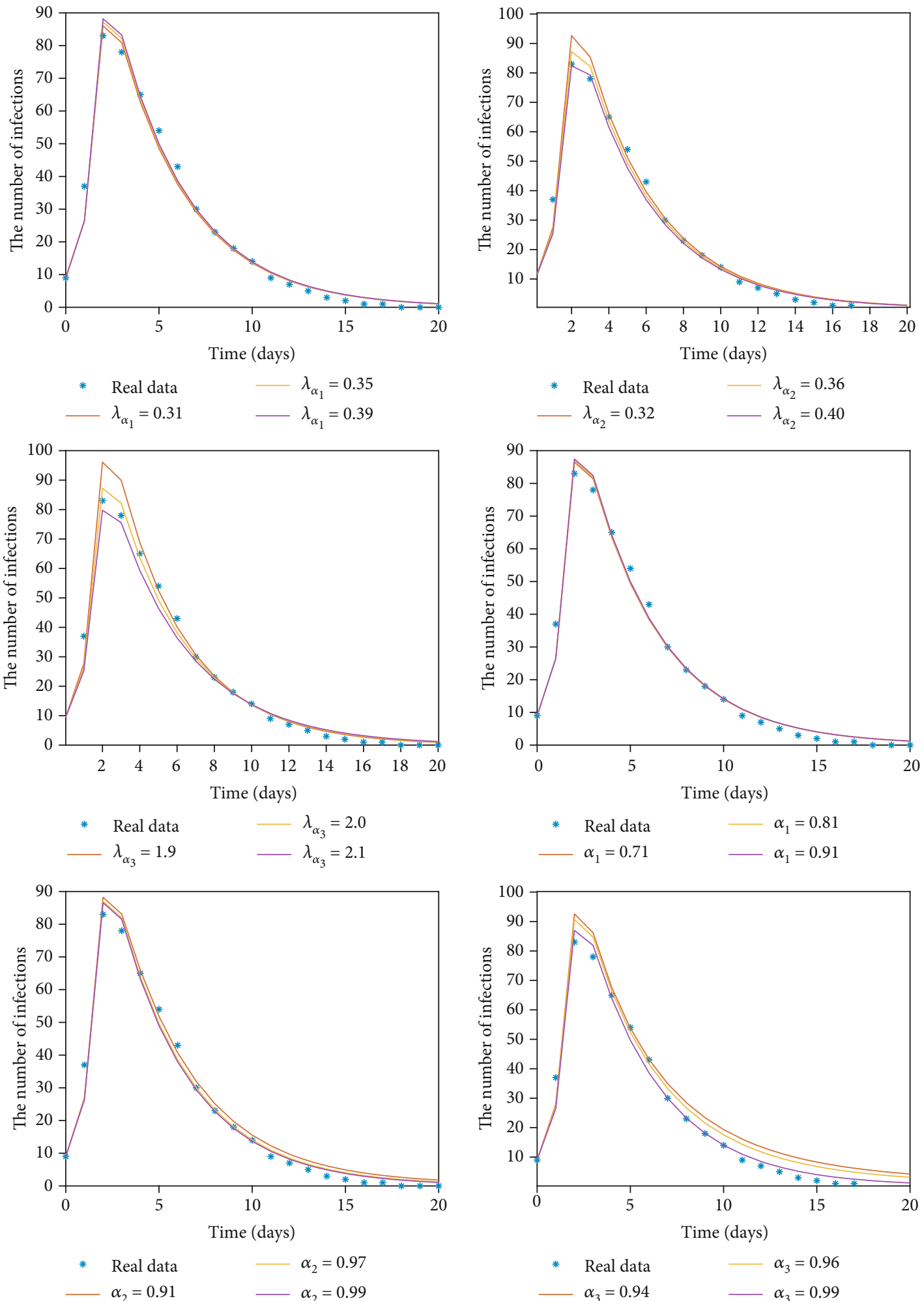

FIGURE 3: The influences of $\lambda_{\alpha_{i}}, \alpha_{i}(i=1,2,3)$ on the quantity of infections $I(t)$ when the remaining parameters remain unchanged. 

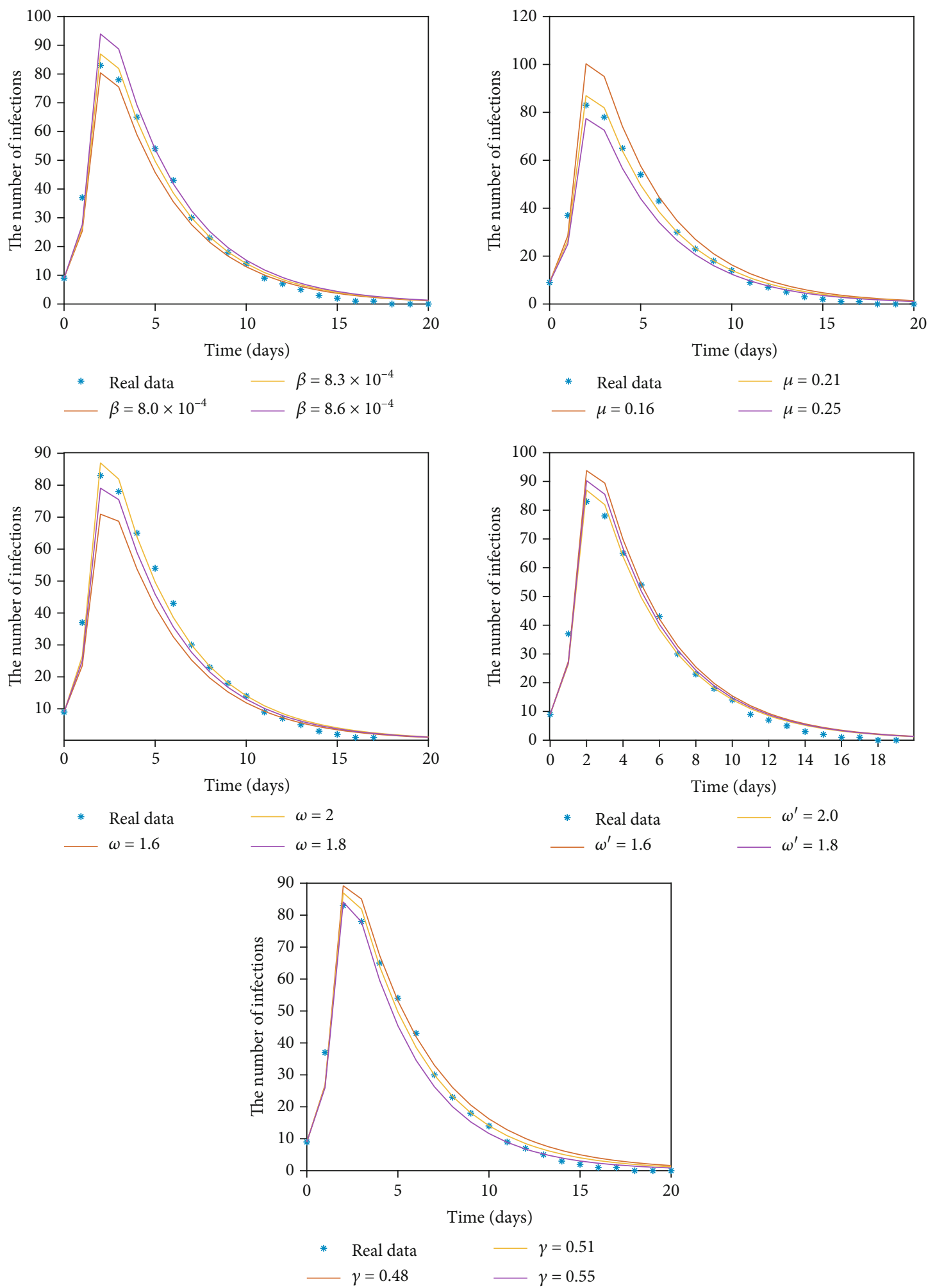

FIGURE 4: The influences of $\beta, \mu, \omega, \omega^{\prime}, \gamma$ on the quantity of infections $I(t)$ when the remaining parameters remain unchanged. 
It follows from equation (67) that

$$
\sum_{k=0}^{n} B_{k}^{n}\left(\mathbf{x}\left(t_{n-k}\right)-\mathbf{x}(0)\right)+\frac{1}{h}\left(\mathbf{x}\left(t_{n}\right)-\mathbf{x}\left(t_{n-1}\right)\right)=\mathbf{f}\left(t_{n}, \mathbf{u}\left(t_{n}\right)\right),
$$

i.e.,

$$
\begin{aligned}
\mathbf{x}\left(t_{n}\right)= & \frac{\mathbf{f}\left(t_{n}, \mathbf{x}\left(t_{n}\right)\right)}{B_{0}^{n}+h^{-1}}+\frac{B_{0}^{n} \mathbf{x}(0)+h^{-1} \mathbf{x}\left(t_{n-1}\right)}{B_{0}^{n}+h^{-1}} \\
& -\sum_{k=1}^{n} \frac{B_{k}^{n}}{B_{0}^{n}+h^{-1}}\left(\mathbf{x}\left(t_{n-k}\right)-\mathbf{x}(0)\right) .
\end{aligned}
$$

Since there is an unknown variable $\mathbf{x}\left(t_{n}\right)$ on both sides of (69), we choose the Newton method to gain the value of $\mathbf{x}\left(t_{n}\right)$ via equation (69).

6.2. The Numerical Simulations of the Three-Term Fractional-Order SEIAR Model. In the following, we use the above GMMP scheme $[46,47]$ to exhibit the numerical solution for simulating this SEIAR model (52) and also utilize the MH-NMSS-PSO algorithm to find the ideal fractional orders and parameters, with which the three-term fractional SEIAR model (52) can provide numerical results that fit well with the real data.

As the one-term SEIAR model (16), each of the parameters in the multiterm SEIAR model (52) has its particular biological meaning. And each parameter has a corresponding value range. Then, each parameter has a corresponding value range. The choice of parameter intervals to narrow the target value is crucial for the result. Hence, based on these ranges, the intervals and initial velocities are chose in this way:

$$
\begin{aligned}
& 0 \leq \lambda_{1}=p_{1} \leq 2, \quad 0 \leq \lambda_{2}=p_{2} \leq 2, \quad 0 \leq \lambda_{0}=p_{3} \leq 2, \\
& 0 \leq \alpha_{1}=p_{4} \leq 1, \quad 0 \leq \alpha_{2}=p_{5} \leq 2, \quad 1 \times 10^{-4} \leq \beta=p_{6} \leq 1 \times 10^{-3}, \\
& 1 \times 10^{-11} \leq k=p_{7} \leq 1 \times 10^{-10}, \quad 0.01 \leq \mu=p_{8} \leq 0.5, \quad 0.5 \leq \omega=p_{9} \leq 2, \\
& 0.5 \leq \omega^{\prime}=p_{10} \leq 2, \quad \frac{1}{6} \leq \gamma=p_{11} \leq 1, \quad 0.01 \leq \gamma^{\prime}=p_{12} \leq 0.092, \\
& V_{i}= \begin{cases}0.01, & i=1,2, \cdots, 5, \\
1 \times 10^{-4}, & i=6, \\
0.02, & i=8, \\
0.05, & i=9,10, \\
0.02, & i=11, \\
0.01, & i=12 .\end{cases}
\end{aligned}
$$

Based on the potential global minimum of $g(\mathbf{P})$, the unknown parameter $\mathbf{P}^{*}$ is obtained:

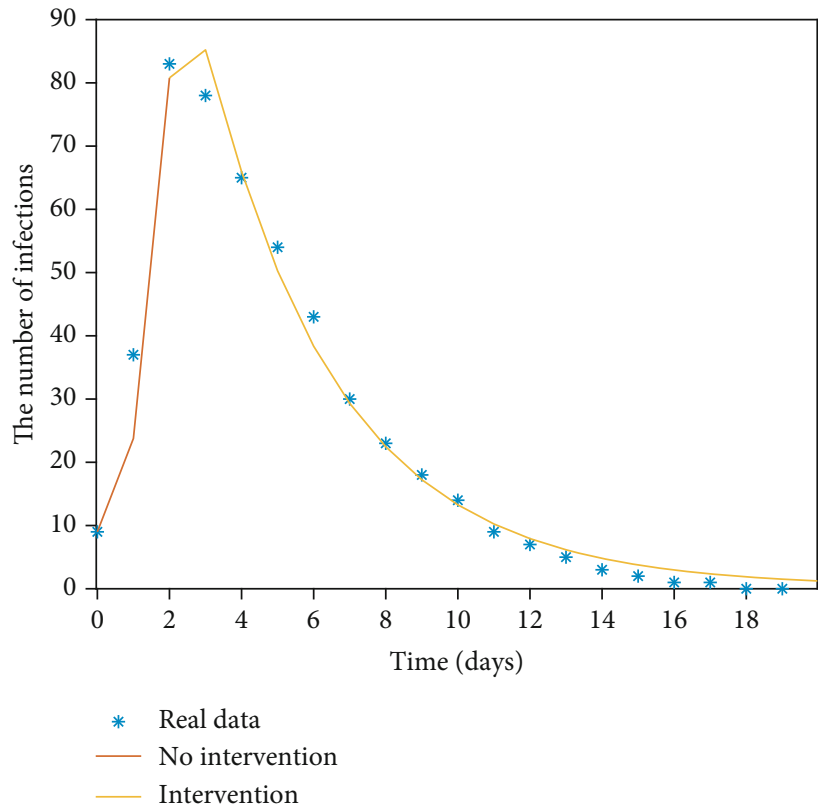

FIgURE 5: Quantity of the Norovirus infections $I(t)$ compared with the numerical results of the SEIAR model (52) obtained by MGAM method and $g=3.7399$.

$$
\begin{gathered}
\lambda_{1}=0.32333, \quad \lambda_{2}=0.000346, \quad \lambda_{0}=0.27650, \quad \alpha_{1}=0.98135, \\
\alpha_{2}=0.99672, \quad \beta=4.3321 \times 10^{-4}, \quad \kappa=1.0035 \times 10^{-11}, \quad \mu=0.50000, \\
\omega=2.00000, \quad \omega=2.00000, \quad \gamma=0.16667, \quad \gamma^{\prime}=0.16667,
\end{gathered}
$$

with $g=3.7399$. As seen in Figure 5, the three-term fractional SEIAR model (52) can provide numerical results that agree well with the real data.

By observing how the parameters influence the variety of infected people $I(t)$ when the remaining parameters are unchanged, we can obtain the conclusion when the parameters $\lambda_{2}, \alpha_{2}, k, \gamma^{\prime}$ changed; the number of infected people $I(t)$ basically did not change while other parameters have a certain impact on $I(t)$. And the influences of other orders and parameters are given in Figures 6 and 7, which show that the parameter $\mathbf{P}^{*}$ we estimated is indeed the ideal parameter.

In the following, we will study the effects of the isolation which is taken on different days. In reference [42], they study the data about a Norovirus infectious diarrhea incident reported in a middle school in a city. On March 8th, the department of the centers for disease control and prevention of a city received a telephone report from a middle school, saying that there were more than ten cases of vomiting, abdominal pain, and diarrhea in the school recently. The following case definitions were established: vomiting or diarrhea and other symptoms such as abdominal pain, fever, headache, and dizziness have occurred among the students and staff of the school since March 5th. The number of cases reached a peak on March 8th. After the intervention on March 8th, isolation measures were taken. The epidemic 

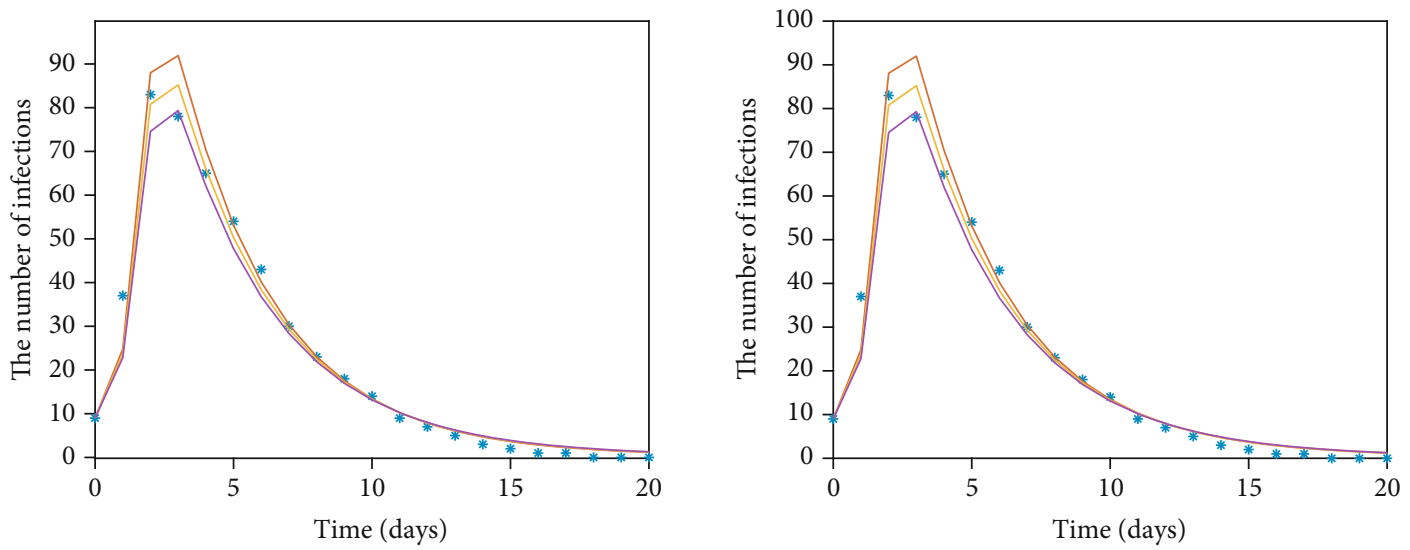

* Real data $\quad \lambda_{1}=0.32$

$\lambda_{1}=0.30 \quad-\lambda_{1}=0.34$

* Real data $\quad \lambda_{0}=0.27$

$\lambda_{0}=0.25 \quad-\lambda_{0}=0.29$
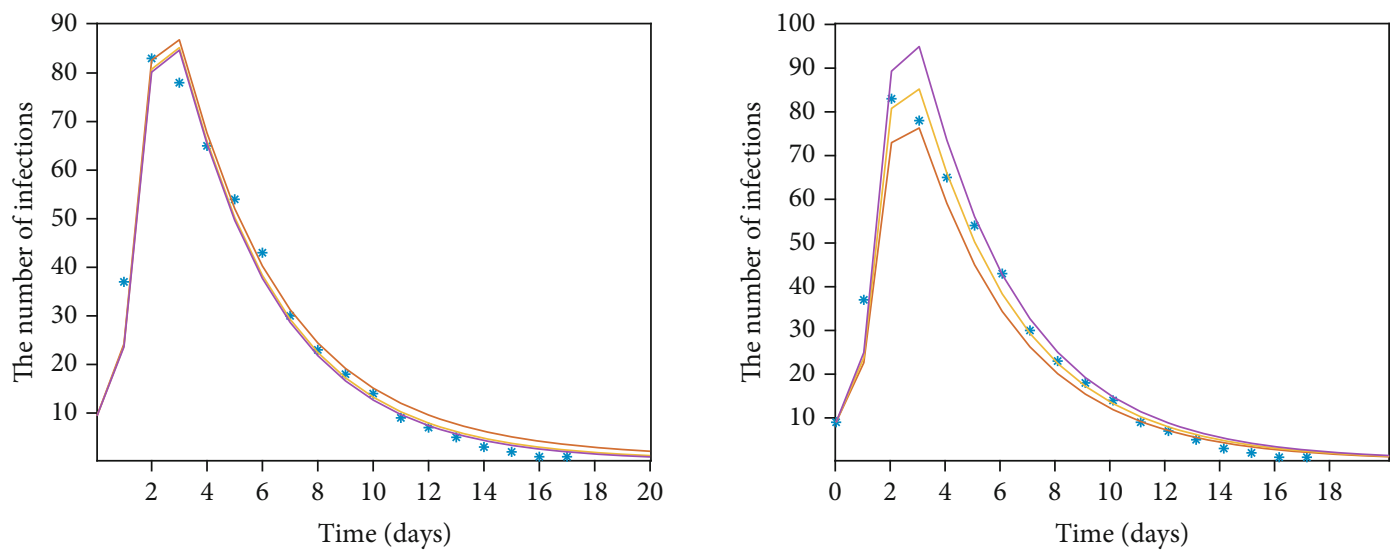

* Real data $\quad \alpha_{1}=0.98$

$-\alpha_{1}=0.95-\alpha_{1}=0.99$
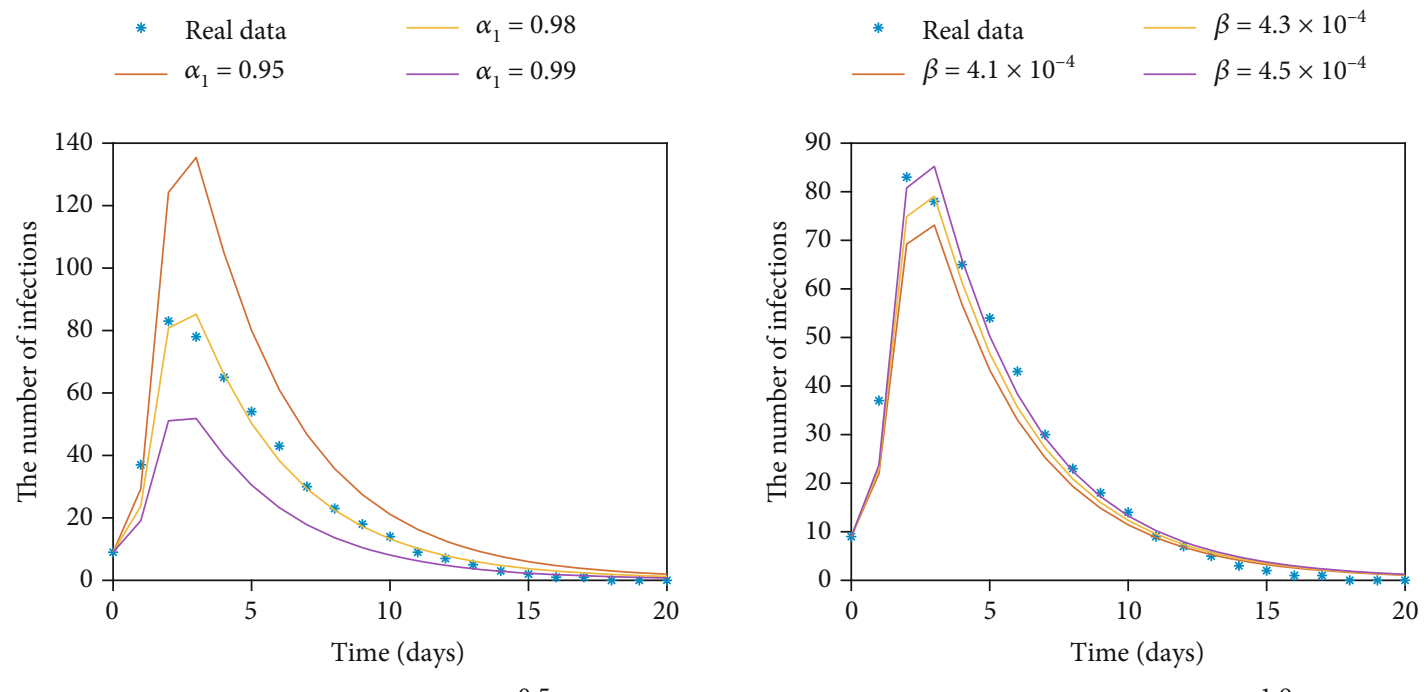

* Real data $\quad \mu=0.5$

* Real data $\quad \omega=1.9$

FIGURE 6: The influences of $\lambda_{1}, \lambda_{0}, \alpha_{1}, \beta, \mu, \omega$ on the quantity of infections $I(t)$ when the remaining parameters remain unchanged.

situation began to decline gradually. In the following, we will assess the effects of the isolation on different days. From the numerical simulation and Figure 8 , the conclusion about the effects of isolation can be obtained in the following. When the intervention and isolation measures were taken on March 8 th, the number of infected people reached a peak on March 8th. When the isolation measure were taken after March 8th, the number of the infected people would increase more than the real data. In particular, when the isolation measures were taken on March 12th, the changes of the number of the infected people would be similar to those with no isolation measure, which indicates that the intervention and isolation 

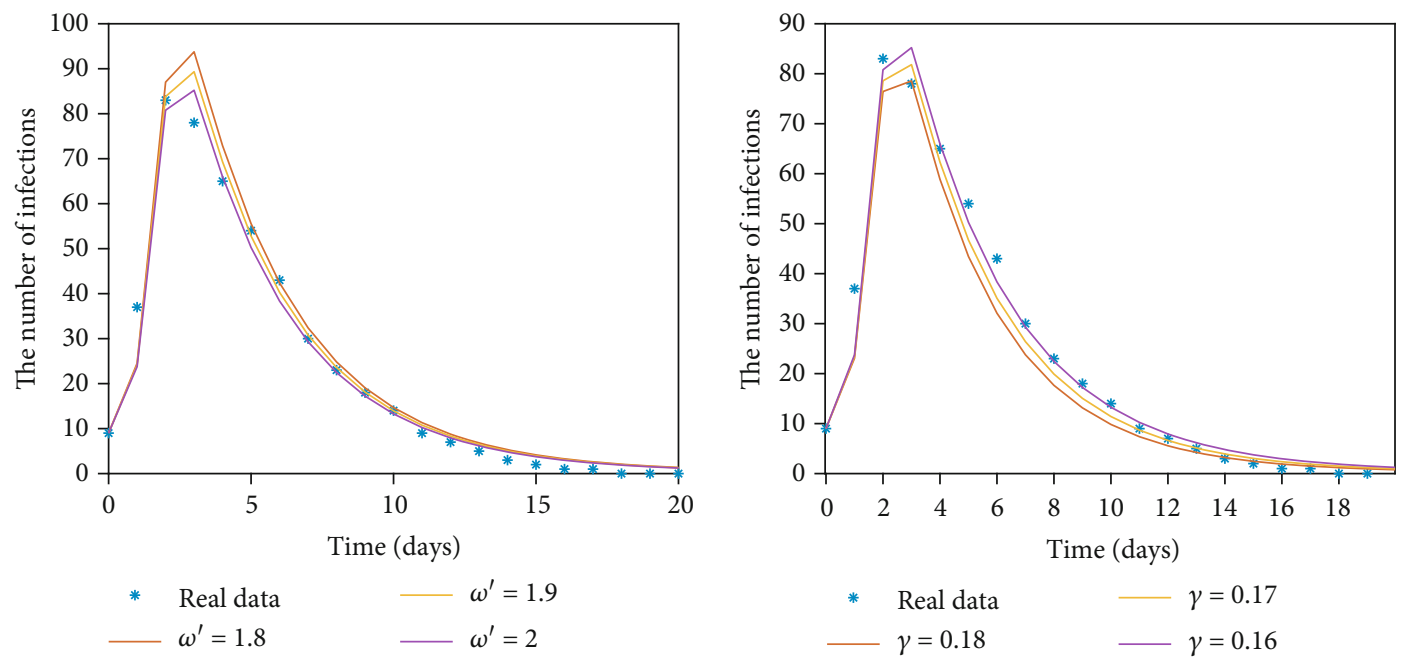

FIGURE 7: The influences of $\omega^{\prime}, \gamma$ on the quantity of infections $I(t)$ when the remaining parameters remain fixed.

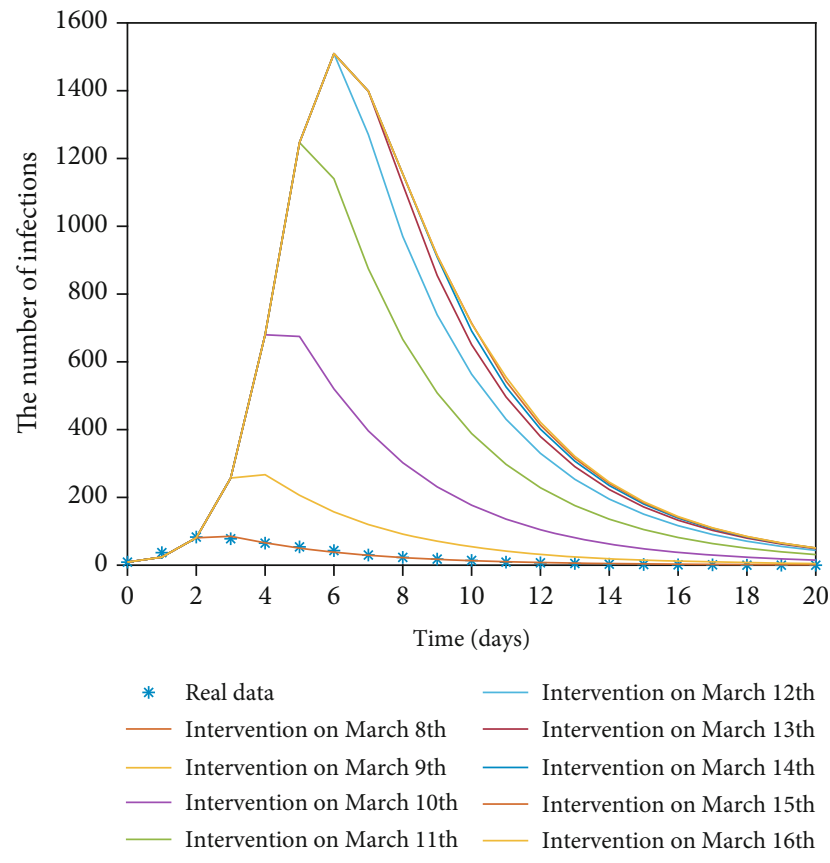

FIgURE 8: Changes in the number of the infections $I(t)$ of the multiterm fractional-order SEIAR model when the intervention and isolation are taken on different days.

are ineffective for controlling the number of the infected people. These conclusions have practical significance for controlling the spread of the Norovirus. The earlier the intervention and isolation are taken, the less people are infected.

\section{Conclusion}

In this research work, we devoted to studying general and multiterm fractional-order SEIAR models considering the Caputo fractional derivative. And we also research the inverse problem of parameter estimation for the fractionalorder SEIAR systems. To derive the numerical solutions of the fractional-order SEIAR differential equation, the GMMP scheme and Newton method are introduced. Simultaneously, the way we estimate orders and parameters is by applying the MH-NMSS-PSO algorithm. To guarantee the correctness and effectiveness of the proposed methods, the data of a 2007 Norovirus outbreak in a middle school [42] is used as the real data to conceive the parameter estimation. With the new parameters, all figures demonstrate that the numerical solutions fit very well with the real data, which prove whether the single-term or the multiterm fractional-order SEIAR models can forecast the quantity of the infectious people accurately. And it also verifies the effectiveness of our method for parameter estimation. And the impact of every parameter on the quantity of infected humans $I(t)$ is studied with the remaining parameters unchanged. The result shows that the parameters we got are indeed the most ideal parameters, and the fractional-order SEIAR model we proposed can provide a better fitting among the numerical solutions and the real data than other models.

The hybrid Nelder-Mead simplex search and particle swarm optimization (MH-NMSS-PSO) algorithms are a valid method of estimating the fractional derivative orders and coefficients for fractional differential equations. In the paper [58], they propose a feasible parameter estimation method based on a hybrid Nelder-Mead simplex search and particle swarm optimization to perform the curve fitting for the multiterm time-fractional Bloch equations. From the simulated results, shown in Figure 5 of their paper, the numerical results of the two-term TFBE model (solid line) are in good agreement with the experimental data (asterisk), which shows that the parameter estimation method $(\mathrm{MH}-$ NMSS-PSO) is effective. In the paper [10], they apply a parameter fitting approach to the classical monoexponential model, a previously developed anomalous relaxation model, and the extended time-fractional relaxation model. They find that the extended time-fractional model is able to fit the experimental data with smaller mean-squared error than the classical monoexponential relaxation model and the anomalous relaxation model, which is shown in Figure 2. Other papers about the inverse problem also show that the 
MH-NMSS-PSO algorithm is an efficient and valid method of estimating parameters for fractional differential equations.

In our paper, we use the modified hybrid Nelder-Mead simplex search and particle swarm optimization $(\mathrm{MH}-$ NMSS-PSO) algorithm to estimate the parameters for fractional differential equations and the multiterm fractional differential equations. The modified MH-NMSS-PSO algorithm is more efficient than the classical one. With the new parameters, our multiterm fractional-order SEIAR system is capable of providing numerical results that agree very well with the real data. It also demonstrates that our dengue model can predict the number of infected human with other data, and our method can be applied to other fractional-order epidemic models.

\section{Data Availability}

The data used to support the findings of this study are available from the corresponding author upon request.

\section{Conflicts of Interest}

The authors declare that they have no competing interests.

\section{Acknowledgments}

This work is partly supported by the Sichuan Science and Technology Foundation (Grant No. 2019YJ0456), the fund of Education Department of Sichuan Province (Grant No. 18ZA0342), and the fund of Sichuan University of Science and Engineering (Grant Nos. 2020RC26 and 2020RC42).

\section{References}

[1] M. de Wit, M. Koopmans, L. Kortbeek et al., "Sensor, a population-based cohort study on gastroenteritis in the Netherlands: incidence and etiology," American Journal of Epidemiology, vol. 154, no. 7, pp. 666-674, 2001.

[2] E. Scallan, R. Hoekstra, F. Angulo et al., "Foodborne illness acquired in the United States-major pathogens," Emerging Infectious Diseases, vol. 17, no. 1, pp. 7-15, 2011.

[3] M. Patel, M. Widdowson, R. Glass, K. Akazawa, J. Vinjé, and U. D. Parashar, "Systematic literature review of role of noroviruses in sporadic gastroenteritis," Emerging Infectious Diseases, vol. 14, no. 8, pp. 1224-1231, 2008.

[4] B. Lopman, A. Hall, A. Curns, and U. D. Parashar, "Increasing rates of gastroenteritis hospital discharges in US adults and the contribution of norovirus, 1996-2007," Clinical Infectious Diseases, vol. 52, no. 4, pp. 466-474, 2011.

[5] A. Hall, A. Curns, L. McDonald, U. D. Parashar, and B. A. Lopman, "The roles of Clostridium difficile and norovirus among gastroenteritis-associated deaths in the United States, 1999-2007," Clinical Infectious Diseases, vol. 55, no. 2, pp. 216-223, 2012.

[6] B. Lopman, G. Adak, M. Reacher, and D. W. G. Brown, "Two epidemiologic patterns of norovirus outbreaks: surveillance in England and Wales, 1992-2000," Emerging Infectious Diseases, vol. 9, no. 1, pp. 71-77, 2003.

[7] P. Teunis, C. Moe, P. Liu et al., "Norwalk virus: how infectious is it?," Journal of Medical Virology, vol. 80, no. 8, pp. 14681476, 2008.
[8] T. Chen, R. Leung, Z. Zhou, R. Liu, X. Zhang, and L. Zhang, "Investigation of key interventions for shigellosis outbreak control in China," PLoS One, vol. 9, no. 4, article e95006, 2014.

[9] T. Chen, R. Ka-Kit Leung, R. Liu et al., "Risk of imported Ebola virus disease in China," Travel Medicine and Infectious Disease, vol. 12, 6 Part A, pp. 650-658, 2014.

[10] S. Qin, F. Liu, I. W. Turner, Q. Yu, Q. Yang, and V. Vegh, "Characterization of anomalous relaxation using the timefractional Bloch equation and multiple echo $\mathrm{T}_{2} *$-weighted magnetic resonance imaging at 7 T," Magnetic Resonance in Medicine, vol. 77, no. 4, pp. 1485-1494, 2017.

[11] F. Liu, V. Anh, and I. Turner, "Numerical solution of the space fractional Fokker-Planck equation," Journal of Computational and Applied Mathematics, vol. 166, no. 1, pp. 209-219, 2004.

[12] R. L. Magin, "Fractional calculus models of complex dynamics in biological tissues," Computers \& Mathematics with Applications, vol. 59, no. 5, pp. 1586-1593, 2010.

[13] X. Jiang and H. Qi, “Thermal wave model of bioheat transfer with modified Riemann-Liouville fractional derivative," Journal of Physics A: Mathematical and Theoretical, vol. 45, no. 48, article 485101, 2012.

[14] I. Podlubny, Fractional Differential Equations, Academic, New York, 1999.

[15] I. Petras, Fractional-order nonlinear systems: modeling, analysis and simulation, Higher Education Press, Beijing, 2011.

[16] Y. Ding, Z. Wang, and H. Ye, "Optimal control of a fractionalorder HIV-immune system with memory," IEEE Transactions on Control Systems Technology, vol. 99, pp. 1-7, 2011.

[17] M. el-Shahed and A. Alsaedi, "The fractional SIRC model and influenza A," Mathematical Problems in Engineering, vol. 2011, Article ID 480378, 9 pages, 2011.

[18] E. Hanert, E. Schumacher, and E. Deleersnijder, "Front dynamics in fractional-order epidemic models," Journal of Theoretical Biology, vol. 279, no. 1, pp. 9-16, 2011.

[19] K. Diethelm, N. J. Ford, A. D. Freed, and Y. Luchko, “Algorithms for the fractional calculus: a selection of numerical methods," Computer Methods in Applied Mechanics and Engineering, vol. 194, no. 6-8, pp. 743-773, 2005.

[20] H. Zhang, X. Jiang, and X. Yang, "A time-space spectral method for the time-space fractional Fokker-Planck equation and its inverse problem," Applied Mathematics and Computation, vol. 320, pp. 302-318, 2018.

[21] T. Feng, M. Gulliksson, and W. Liu, "Adaptive finite element methods for the identification of elastic constants," Journal of Scientific Computing, vol. 26, no. 2, pp. 217-235, 2006.

[22] P. H. Kloppers and J. C. Greeff, "Lotka-Volterra model parameter estimation using experiential data," Applied Mathematics and Computation, vol. 224, pp. 817-825, 2013.

[23] L. Fermo, C. van der Mee, and S. Seatzu, "Parameter estimation of monomial-exponential sums in one and two variables," Applied Mathematics and Computation, vol. 258, pp. 576-586, 2015.

[24] B. Yu, X. Jiang, and H. Xu, "A novel compact numerical method for solving the two-dimensional non-linear fractional reaction-subdiffusion equation," Numerical Algorithms, vol. 68, no. 4, pp. 923-950, 2015.

[25] Y. Li, X. Meng, B. Zheng, and Y. Ding, "Parameter identification of fractional order linear system based on Haar wavelet operational matrix," ISA Transactions, vol. 59, pp. 79-84, 2015. 
[26] J. Cheng, J. Nakagawa, M. Yamamoto, and T. Yamazaki, "Uniqueness in an inverse problem for a one-dimensional fractional diffusion equation," Inverse Problems, vol. 25, no. 11, p. 115002, 2009.

[27] S. Qin, F. Liu, I. Turner, V. Vegh, Q. Yu, and Q. Yang, "Multi-term time-fractional Bloch equations and application in magnetic resonance imaging," Journal of Computational and Applied Mathematics, vol. 319, pp. 308-319, 2017.

[28] W. Fan, X. Jiang, and H. Qi, "Parameter estimation for the generalized fractional element network Zener model based on the Bayesian method," Physica A: Statistical Mechanics and its Applications, vol. 427, pp. 40-49, 2015.

[29] T. Wei and J. Wang, "A modified quasi-boundary value method for an inverse source problem of the time-fractional diffusion equation," Applied Numerical Mathematics, vol. 78, pp. 95-111, 2014.

[30] N. Jain, S. Jhunthra, H. Garg et al., "Prediction modelling of COVID using machine learning methods from B-cell dataset," Results in Physics, vol. 21, p. 103813, 2021.

[31] N. Ghorui, A. Ghosh, S. P. Mondal et al., "Identification of dominant risk factor involved in spread of COVID-19 using hesitant fuzzy MCDM methodology," Results in Physics, vol. 21, p. 103811, 2021.

[32] M. Zamir, K. Shah, F. Nadeem et al., "Threshold conditions for global stability of disease free state of COVID-19," Results in Physics, vol. 21, no. 1, p. 103784, 2021.

[33] A. Raza, A. Ahmadian, M. Rafiq, S. Salahshour, and M. Ferrara, "An analysis of a nonlinear susceptible-exposedinfected-quarantine-recovered pandemic model of a novel coronavirus with delay effect," Results in Physics, vol. 21, p. 103771, 2021.

[34] P. Veeresha, H. M. Baskonus, D. G. Prakasha, W. Gao, and G. Yel, "Regarding new numerical solution of fractional Schistosomiasis disease arising in biological phenomena," Chaos, Solitons and Fractals, vol. 133, p. 109661, 2020.

[35] W. Gao, P. Veeresha, H. M. Baskonus, D. G. Prakasha, and P. Kumar, "A new study of unreported cases of 2019-nCOV epidemic outbreaks," Chaos, Solitons and Fractals, vol. 138, p. 109929, 2020.

[36] S. Rezapour, S. Etemad, and H. Mohammadi, "A mathematical analysis of a system of Caputo-Fabrizio fractional differential equations for the anthrax disease model in animals," Advances in Difference Equations, vol. 2020, no. 1, Article ID 481, 2020.

[37] D. Baleanu, A. Jajarmi, H. Mohammadi, and S. Rezapour, "A new study on the mathematical modelling of human liver with Caputo-Fabrizio fractional derivative," Chaos. Solitons Fractals, vol. 134, p. 109705, 2020.

[38] I. Ameen, D. Baleanu, and H. M. Ali, “An efficient algorithm for solving the fractional optimal control of SIRV epidemic model with a combination of vaccination and treatment," Chaos. Solitons Fractals, vol. 137, p. 109892, 2020.

[39] B. Shiri, G. C. Wu, and D. Baleanu, "Collocation methods for terminal value problems of tempered fractional differential equations," Applied Numerical Mathematics, vol. 156, pp. 385-395, 2020.

[40] W. Gao, H. M. Baskonus, and L. Shi, "New investigation of bats-hosts-reservoir-people coronavirus model and application to 2019-nCoV system," Advances in Difference Equations, vol. 2020, no. 1, Article ID 391, 2020.
[41] W. Gao, P. Veeresha, D. G. Prakasha, and H. M. Baskonus, "Novel dynamic structures of 2019-nCoV with nonlocal operator via powerful computational technique," Biology, vol. 9, no. 5 , p. $107,2020$.

[42] "Is there a population awareness of microbes: the dynamics of Norovirus transmission," CTModelling, 2018, https://mp .weixin.qq.com/s/gMdemZE1Vl1r4DHuDqPoQw.

[43] F. Wang, Y. Zhang, C. Wang, and J. Ma, "Stability analysis of an $e$-SEIAR model with point-to-group worm propagation," Communications in Nonlinear Science and Numerical Simulation, vol. 20, no. 3, pp. 897-904, 2015.

[44] K. Diethelm, The Analysis of Fractional Differential Equations, vol. 9, Springer, Berlin Heidelberg, 2004.

[45] T. Li, Y. Wang, and M. Luo, "Control of chaotic and hyperchaotic systems based on a fractional order controller," Chinese Physics B, vol. 23, pp. 0805011-08050111, 2014.

[46] R. Gorenflo, F. Mainardi, D. Moretti, and P. Paradisi, "Time fractional diffusion: a discrete random walk approach," Nonlinear Dynamics, vol. 29, no. 1/4, pp. 129-143, 2002.

[47] J. Quintana Murillo and S. Bravo Yuste, "On three explicit difference schemes for fractional diffusion and diffusionwave equations," Physica Scripta, vol. T136, p. 014025, 2009.

[48] J. A. Nelder and R. Mead, "A simplex method for function minimization," The Computer Journal, vol. 7, no. 4, pp. 308313, 1965.

[49] R. C. Eberhart and J. Kennedy, "A new optimizer using particle swarm theory," in Proceedings of the sixth international symposium on micro machine and human science, vol. 1, pp. 39-43, New York, NY, 1995.

[50] N. F. A. Hamid, N. A. Rahim, and J. Selvaraj, "Solar cell parameters identification using hybrid Nelder-Mead and modified particle swarm optimization," Journal of Renewable and Sustainable Energy, vol. 8, no. 1, p. 015502, 2016.

[51] S. H. Liao, J. G. Hsieh, J. Y. Chang, and C. T. Lin, "Training neural networks via simplified hybrid algorithm mixing Nelder-Mead and particle swarm optimization methods," Soft Computing, vol. 19, no. 3, pp. 679-689, 2015.

[52] T. Li, Y. Wang, F. Liu, and I. Turner, "Novel parameter estimation techniques for a multi-term fractional dynamical epidemic model of dengue fever," Numerical Algorithms, vol. 82, no. 4, pp. 1467-1495, 2019.

[53] N. F. Hussam, H. Amjad, and S. Azzam, "Hybrid particle swarm optimization with sine cosine algorithm and NelderMead simplex for solving engineering design problems," Arabian Journal for Science and Engineering, vol. 45, no. 11, pp. 3091-3109, 2020.

[54] E. Zahara and Y. T. Kao, "Hybrid Nelder-Mead simplex search and particle swarm optimization for constrained engineering design problems," Expert Systems with Applications, vol. 36 , no. 2, pp. 3880-3886, 2008.

[55] H. N. Fakhouri, A. Hudaib, and A. Sleit, "Hybrid particle swarm optimization with sine cosine algorithm and NelderMead simplex for solving engineering design problems," Arabian Journal for Science and Engineering, vol. 45, no. 4, pp. 3091-3109, 2020.

[56] Y. S. Zhang, C. J. Dai, B. Wang, and Z. Y. Zhu, "Viewpoint selection using hybrid simplex search and particle swarm optimization for volume rendering," International Journal of Image, Graphics and Signal Processing (IJIGSP), vol. 4, no. 9, pp. 17-22, 2012. 
[57] F. Barzinpour, R. Noorossana, S. T. A. Niaki, and M. J. Ershadi, "A hybrid Nelder-Mead simplex and PSO approach on economic and economic-statistical designs of MEWMA control charts," The International Journal of Advanced Manufacturing Technology, vol. 65, no. 9-12, pp. 1339-1348, 2013.

[58] S. Qin, F. Liu, I. Turner, V. Vegh, Q. Yu, and Q. Yang, "Multiterm time-fractional Bloch equations and application in magnetic resonance imaging," Journal of Computational and Applied Mathematics, vol. 319, pp. 308-319, 2017. 